\title{
Percepción del Personal de Escuelas Municipales Sobre Abuso Sexual Infantil: Estudio Exploratorio
}

\section{Perception of Municipal School Personnel about Child Sexual Abuse: A Exploratory Study}

\author{
PSI. JuAn Díaz URRea (D) \\ Facultad de Ciencias Sociales y Comunicaciones, Universidad Santo Tomás, Antofagasta, Chile. \\ Psi. Sebastián Araya Cuevas (iD \\ Facultad de Ciencias Sociales y Comunicaciones, Universidad Santo Tomás, Antofagasta, Chile.

\section{Psi. María de los Angeles Valenzuela Méndez [0} \\ Facultad de Ciencias Sociales y Comunicaciones, Universidad Santo Tomás, Antofagasta, Chile.

\section{Mg. Karina del Pilar Tejada Campos (1)} \\ Facultad de Ciencias Sociales y Comunicaciones, Universidad Santo Tomás, Antofagasta, Chile.
}

Autor de correspondencia: Psi. María de los Angeles Valenzuela Méndez

email: anyeles.am@gmail.com

\section{RESUMEN}

El Abuso Sexual Infantil (ASI) deja consecuencias psicológicas profundas, su prevalencia llega casi al 30\% a nivel mundial. En Chile los infantes entre 8 y 13 años pasan 1.100 horas efectivas en aula, siendo la escuela fundamental para la protección, prevención y detección del ASI. Objetivo: Establecer la percepción sobre ASI de funcionarios de escuelas municipales en la comuna de Antofagasta. Método: Cuantitativo; entre los instrumentos aplicados se utilizó cuestionario de Márquez, M. et al., 2016; contó con 703 trabajadores de 20 escuelas. Resultados: la mayoría posee un conocimiento teórico básico, aunque un $67,8 \%$ no recibe capacitaciones; un $70 \%$ señaló conocer a una víctima de ASI, pero de estos el 77,2\% no ha realizado denuncias; un 71,4\% creen erróneamente que el agresor tiene características patológicas evidentes. El equipo del Programa de Integración Escolar, de entre los profesionales que atienden directamente infantes, apenas un $25 \%$ conoce métodos de evaluación de ASI, sólo el 55,3\% reconoce la posibilidad de abuso en un infante, un 81\% declara conocer víctimas, pero un escaso $22 \%$ ha denunciado. Estos resultados muestran la probable invisibilización del fenómeno, aumentando el riesgo para los infantes, entre ellos a los más vulnerables, y por tanto la necesidad de intervenciones urgentes.

Palabras Clave: Abuso Sexual Infantil, Contexto Educativo, Agentes Educativos, Establecimientos Educacionales. 


\section{ABSTRACT}

The Child Sexual Abuse (CSA) leaves profound psychological consequences, its prevalence reaches almost $30 \%$ worldwide. In Chile, infants between 8 and 13 years spend 1.100 effective hours in the classroom, being the school fundamental for protection, prevention and detection of CSA. Objective: Establish the perception about CSA in personnel of municipal schools in Antofagasta district. Method: Quantitative; among the applied instruments, a questionnaire from Márquez, M. et al., 2016; presented an amount of 703 workers from 20 schools. Results: the majority have a basic knowledge, although $67,8 \%$ don>t receive training; $70 \%$ said they knew a victim of CSA, but of these $77,2 \%$ had not made any denounce; $71,4 \%$ mistakenly believe that the aggressor has obvious pathological characteristics. The School Prevention of Integration program, among workers who directly care for infants, only 25\% knows CSA assessment methods, only 55,3\% recognize the possibility of abuse in an infant, $81 \%$ declare they know victims, but a scarce $22 \%$ have denounced. The results show the probable invisibility of the phenomenon, increasing the risk for infants, including the most vulnerable, and therefore the need for urgent interventions.

Keywords: Child Sexual Abuse, Educational Context, Educative Agents, Educational Establishments.

\section{Introducción}

Delitos de connotación física y psicológica han sido parte del desarrollo social durante siglos, incluyendo las violaciones hacia los infantes y el Abuso Sexual Infantil (ASI) (Baita y Moreno, 2015). La prevalencia mundial sobre víctimas de ASI para el 2017, según el Fondo de las Naciones Unidas (UNICEF), es de 15 millones de mujeres, entre los 15 y 19 años. Así mismo, en 28 países estudiados, 9 de cada 10 niñas son obligadas a relacionarse sexualmente con algún conocido o familiar. En Chile, conforme al Servicio Nacional de Menores (SENAME) (2014), de 43.929 sujetos ingresados por maltrato y abuso sexual, el 21\% fue por ASI. Por otra parte, la Fiscalía Nacional 2018, desglosa que las cifras de delitos sexuales comprenden un total de 28.132 denuncias, de las cuales el 25,35\% comprende abuso sexual y el $22,9 \%$ violaciones, siendo el $71,36 \%$ de las víctimas, menores de edad.

ASI se define como la tocación indebida, insinuación, acoso, exposición infantil y/o prostitución de un menor de edad, dada mediante una asimetría de poder (ya sea de edad o tamaño), entre víctima y victimario; donde la víctima sufre de consecuencias físicas y/o psicológicas, tanto a corto como a largo plazo, existiendo además, distintos factores de riesgo sociales, familiares y personales (Cantón-Cortés, 2013; Cantón-Cortés y Rosario, 2012; Giménez y Valladolid, 2017; Villanueva, 2013; Pereda y Sicilia, 2015; Franco y Ramírez, 2015; Manzi y Asensio, 2015; Pereda y Abad, 2012; Apraez, 2015; Bravo y Meléndez, 2016; González y Carrasco, 2016).

En la sociedad contemporánea, la falta de investigación respecto de la construcción social de la sexualidad, permite la perpetuación de modelos violentos y desiguales, que propician la aparición del ASI (Franco y Ramírez, 2015), por lo tanto, ante la desinformación los casos de abuso sexual continúan, el porcentaje de víctimas a nivel mundial, entre 1946 y 2011, es de 28,65\% (Losada, 2012). Lo complejo, es que no necesariamente debe haber contacto físico para que sea considerado abuso (Manzi y Asensio, 2015), entonces no necesariamente existen consecuencias físicas, invisibilizando la problemática, volviéndose imperante la develación y el relato (Pereda y Abad, 2012).

La evidencia indica, que la escuela es uno de los agentes socializadores primarios en la vida del infante (Yubero, 2005), en Chile, se cumple un total de 1.100 horas anuales en la educación básica, su- 
perando la media de los países de la OECD (Martinic, 2015), demostrando que los establecimientos tienen el deber de diagnosticar e intervenir, de forma prematura, pudiendo disminuir las consecuencias negativas a largo plazo, (Cantón-Cortes y Rosario, 2015), siendo el personal quien adquiere un papel decisivo ante esta problemática (Rúa, Pérez y González, 2018), situándose como un problema de salud pública, donde deben existir profesionales capacitados y se implementen programas de prevención (Losada, 2012).

En el campo de la investigación científica en Chile, existe ausencia de estudios de similares características y que apunte al mismo tipo de muestra. Los existentes se centran en el fenómeno de la victimología y criminología, además de la evaluación de procedimientos de detección y denuncia, como se demuestra en los estudios de Contreras, Huepe y Navarrete (2019); Pinto-Cortez y Guerra (2019); Gutierrez, et al. (2016); Oyanedel y Ortúzar (2018).

Este estudio buscó establecer la percepción sobre ASI del personal de los establecimientos educacionales, determinando el conocimiento teórico y creencias sobre ASI e identificando percepciones asociadas con el quehacer educativo y aspectos psicológicos.

\section{Método}

El presente, es un estudio de carácter cuantitativo exploratorio, no experimental y medido mediante instrumentos válidos y confiables, con una muestra representativa de la población definida (Rodríguez y Valldeoriola, 2012; Hernández, Fernández y Baptista, 2014).

Este estudio cuenta con la aprobación ética y científica, por parte de la Escuela de Psicología y el Comité de Ética Científico de la Universidad San- to Tomás, y el patrocinio de la Dirección de Educación de la Corporación Municipal de Desarrollo Social de la comuna de Antofagasta, respecto al cual también se realizó devolución de resultados al Programa de Atención Integral del Estudiante.

\section{Población y muestra}

La población contempla al personal de 30 escuelas municipales de Antofagasta, y que cumple con alguno de los siguientes cargos: Docente directivo (DD), administrativos (AD), docente (DC), empleado de servicio, guardia de seguridad, profesional, profesional ss (PSS), proyecto integración (PIE). Según estos criterios, y lo registrado en la base de datos actualizada a octubre del 2018, de la Corporación Municipal de Desarrollo Social (GMDS), la población de estudio es de un total de 2.046 sujetos. La muestra fue de tipo no probabilística y se utilizó un muestreo por conveniencia. Los participantes fueron un total de 703 sujetos de 20 establecimientos educacionales básicos que representaron el $64,5 \%$ de la población. El perfil fue: la mayoría docente (55,1\%); mujeres $(81,1 \%)$; solteras (45,9\%); edad promedio, entre los 19 y 69 años; de nacionalidad chilena $(98,4 \%)$; con estudios superiores completos $(76,1 \%)$ y con 11 años en promedio desempeñándose en educación.

\section{Instrumentos}

\section{Cuestionario Respecto de Conocimiento y Creencias Acerca de ASI.}

Cuestionario Respecto de Conocimiento y Creencias Acerca de ASI, creado por Márquez, Márquez y Granados (2016), quienes adaptaron el cuestionario creado por Pereda et al. (2012), el cual se compone por Escala de Mitos sobre Abu- 
so Sexual Infantil de Collings (1997) y la Escala de Percepción Masculina sobre Abuso Sexual de Nalavany y Abell (2004), añadiendo dos ítems de falsas creencias, en la adaptación de López Sánchez (2014), quedando con un total de 23 preguntas. La versión original del cuestionario fue traducida por el equipo de investigadores, con resguardo a su legitimidad.

\section{Cuestionario de Datos Sociodemográficos.}

Cuestionario cerrado, de respuestas breves, respecto de datos sociodemográficos de los sujetos, elaborado por el equipo de investigación, que contempló: Edad; Sexo; Cargo; Escuela en la que trabaja; Nivel educacional; Título profesional (si aplica); Cantidad de capacitaciones sobre Abuso Sexual Infantil; Años de experiencia laboral en contextos educacionales con menores de edad.

\section{Cuestionario de Control de Sesgo de Selección.}

Cuestionario de elaboración propia de los investigadores, el cual consta de 23 ítems de verdade- ro o falso, evaluando tres aspectos importantes en relación a la temática, conocimiento general, creencias y conocimiento del marco legal chileno en relación al ASI. Este instrumento tiene por objeto, funcionar como placebo, disminuyendo así, el sesgo de selección.

\section{Análisis de datos}

Se llevó a cabo un análisis estadístico descriptivo de las respuestas obtenidas posterior a la aplicación del instrumento, mediante el cálculo de las medidas de tendencia central (Media, Moda y Mediana) obteniendo las frecuencias totales de cada respuesta, y tablas cruzadas, mediante el programa SPSS, realizando una resignificación del análisis estadístico descriptivo, con la evidencia empírica existente.

\section{Resultados}

Respecto a conocimiento teórico y creencias acerca de ASI (todos los cargos, tablas I y II).

Tabla I

Conocimiento Teórico del Personal

\begin{tabular}{|c|c|c|}
\hline Items & Respuesta & \\
\hline P. 1 El criterio principal para definir abuso sexual es & $\begin{array}{l}\text { a. Gualquier acto realizado entre } \\
\text { agresor y víctima, con o sin contacto } \\
\text { físico. }\end{array}$ & $91,00 \%$ \\
\hline $\begin{array}{l}\text { P. } 2 \text { ¿A qué edad crees que normalmente comienzan } \\
\text { los abusos? }\end{array}$ & a. A cualquier edad & $85,30 \%$ \\
\hline $\begin{array}{l}\text { P. } 3 \text { ¿En qué contexto se producen mayoritariamente } \\
\text { los abusos? }\end{array}$ & a. Al interior de la familia & $94,00 \%$ \\
\hline P.4 El abuso sexual ocurre generalmente en & a. Ambos sexos por igual & $81,30 \%$ \\
\hline
\end{tabular}


Items Respuesta

P.5 El género del agresor es c. Ambos sexos por igual

$63,60 \%$

P.6 El porcentaje de las personas que han sido abusa- c. Más del 20\%

$74,30 \%$ das sexualmente es

P.7 ¿Está familiarizado con algún método de evalua- b. No

$75,60 \%$ ción de abuso sexual infantil?

P. ¿Chonoces las consecuencias legales de abusar se- a. Sí

$75,00 \%$ xualmente de un infante?

P.9 Si estuvieras seguro de que ha ocurrido abuso se- a. La importancia del bienestar del 74,00\% xual; lo denunciarías principalmente basado en infante

P.10 Si sólo existieran sospechas de un abuso sexual, a. Sí 86,50\% ¿lo denunciarías a las autoridades?

P.11 La aplicación de programas preventivos sobre a. Sí 76,00\% abuso sexual infantil, ¿serían efectivos?

P.12 ¿Conoces a alguien que haya sido víctima de a. Sí abuso sexual?

Según la tabla I, sobre conocimiento teórico acerca de ASI; el «criterio de definición», «contexto en que se produce», y "porcentaje de víctimas», entre un 74,3\% y un $91 \%$ responde de forma correcta; sobre la «distinción del sexo»; tanto de víctimas como victimarios, y la «edad de inicio de los abusos», entre el $63 \%$ y el $85 \%$ responden de forma incorrecta.
Es destacable que el $76 \%$ piensa que los programas de prevención serían efectivos, y el 75\% conoce sobre las consecuencias legales del abuso sexual, entre un $74 \%$ y $86,5 \%$, denunciarían sólo ante la sospecha y basado en el bienestar del infante, además, el 70\% conoce a alguna víctima, aun cuando el 75,6\% no está familiarizado con algún método de evaluación en la temática.

Tabla II

Creencias del Personal

Items

K.13 El testimonio de un niño es más creíble que el de un adulto

K.14 Hoy en día hay más casos de abuso sexual que antes

K. 15 Si un infante cercano a nosotros sufriera de abuso, lo sabríamos

K.16 Los abusadores de niños no pueden controlar sus impulsos sexuales

K.17 Los abusadores de niños tienen trastornos mentales, son enfermos mentales, o personas con un alto nivel de desequilibrio

K.18 Algunos abusadores tienen la misma edad que la víctima

K.19 Los infantes que han sido víctimas, son responsables de lo que les sucede, ya que pueden prevenirlo

K. 20 A menudo, los infantes inventan haber sido víctimas de abuso sexual, ya que a veces son influenciados por un adulto

\section{Respuesta}

a. Verdadero $83,80 \%$

a. Verdadero $71,80 \%$

a. Verdadero $68,70 \%$

a. Verdadero $63,80 \%$

a. Verdadero $71,40 \%$

b. Falso $\quad 50,60 \%$

b. Falso $\quad 94,80 \%$

b. Falso

$75,30 \%$ 
K.21 Un alto porcentaje de abuso sexual infantil incluye alguna forma de violencia

a. Verdadero $89,50 \%$

$\mathrm{K} .22 \mathrm{El}$ abuso sexual infantil casi siempre ocurre fuera del hogar del infante

b. Falso

$70,70 \%$

K.23 Más de la mitad de los informes sobre abuso sexual infantil son falsos

b. Falso

$84,60 \%$

En la tabla II, respecto a las creencias asociadas al ASI, son positivas cuando, entre un $68,7 \%$ y un $94,8 \%$, identificaría a un infante víctima. Así mismo, el 70,7\% identifica que ocurre frecuentemente dentro del hogar y que más de la mitad de los informes son reales $(84,6 \%)$.

En lo que respecta al relato y a la ocurrencia de este fenómeno, entre el $71,8 \%$ y $89,5 \%$ posee creencias ambiguas, asumiendo que en la actualidad existe una mayor prevalencia de ASI que antes. Finalmente, se observa que, entre un 50,6\% y un $71,4 \%$ considera que el agresor puede no tener la misma edad que la víctima, además de poseer algún tipo de patología mental y carencia para controlar sus impulsos sexuales.

\section{Percepción de aspectos de domino en el} quehacer educativo (cargos DD, AD, DC, PSS, PIE).

Tabla III

\section{Capacitación según cargo}

\begin{tabular}{lrrr}
\hline & ¿Ha recibido capacitación sobre abuso sexual infantil? \\
\hline Docente Directivo & Sí & No & Total \\
Administrativo & $51,8 \%$ & $48,2 \%$ & $100,0 \%$ \\
Docente & $16,9 \%$ & $83,1 \%$ & $100,0 \%$ \\
Empleado de Servicio & $32,4 \%$ & $67,6 \%$ & $100,0 \%$ \\
Guardia de Seguridad & $20,7 \%$ & $79,3 \%$ & $100,0 \%$ \\
Profesional & $0 \%$ & $100,0 \%$ & $100,0 \%$ \\
Profesional SS & $46,4 \%$ & $53,6 \%$ & $100,0 \%$ \\
Programa Integración Escolar & $66,7 \%$ & $33,3 \%$ & $100,0 \%$ \\
\hline
\end{tabular}




\section{Tabla IV}

Dominios de Nivel Educacional

\begin{tabular}{|c|c|c|c|c|c|}
\hline Respuestas & $\begin{array}{l}\text { Directivos } \\
\quad(\%)\end{array}$ & $\begin{array}{l}\text { Administrativos } \\
(\%)\end{array}$ & $\begin{array}{l}\text { Docentes } \\
\quad(\%)\end{array}$ & $\begin{array}{c}\text { Psicosocial } \\
(\%)\end{array}$ & $\begin{array}{l}\mathrm{PIE} \\
(\%)\end{array}$ \\
\hline P.8) ¿Conoces las consecuen- a) Sí & 81,3 & 59,4 & 75,7 & 91,7 & 781,3 \\
\hline $\begin{array}{l}\text { cias legales de abusar sexual- } \\
\text { mente de un infante? }\end{array}$ & 18,7 & 40,6 & 24,3 & 8,3 & 18,7 \\
\hline P.9) Si estuvieras seguro de a) La importancia del & 63,0 & 75,0 & 76,5 & 58,3 & 381,3 \\
\hline $\begin{array}{l}\text { que ha ocurrido abuso se- } \text { b) La obligación mo- } \\
\text { xual; lo denunciarías, princi- } \\
\text { ral y ética }\end{array}$ & 13,0 & 9,4 & 17,4 & 8,3 & 310,4 \\
\hline palmente, basado en: $\quad$ c) La obligación legal & 24,1 & 15,6 & 6,1 & 33,3 & 8,3 \\
\hline $\begin{array}{l}\text { P.10) Si sólo existieran sospe- a) Sí } \\
\text { chas de un abuso sexual, ¿̇o }\end{array}$ & 92,6 & 90,3 & 82,7 & 91,7 & 89,6 \\
\hline $\begin{array}{l}\text { denunciarías a las autorida- b) No } \\
\text { des? }\end{array}$ & 7,4 & 9,7 & 17,3 & 8,3 & 310,4 \\
\hline $\begin{array}{l}\text { P.11) La aplicación de pro- } \\
\text { gramas preventivos sobre }\end{array}$ & 85,2 & 62,3 & 77,6 & 75,0 & 70,8 \\
\hline $\begin{array}{l}\text { abuso sexual infantil, ¿serían b) No } \\
\text { efectivos? }\end{array}$ & 14,8 & 37,7 & 22,4 & 25,0 & 29,2 \\
\hline P.12) ¿Conoces a alguien a) Sí & 78,6 & 66,2 & 73,3 & 83,3 & 31,3 \\
\hline abuso sexual? & 21,4 & 33,8 & 26,7 & 16,7 & 718,8 \\
\hline G. 1) ¿Ha realizado alguna a) Sí & 23,6 & 12,3 & 15,3 & 50,0 & 22,9 \\
\hline $\begin{array}{l}\text { denuncia por abuso sexual b) No } \\
\text { infantil? }\end{array}$ & 76,4 & 87,7 & 84,7 & 50,0 & 77,1 \\
\hline G.2) ¿Ha recibido alguna a) Sí & 51,8 & 16,9 & 32,4 & 66,7 & 733,3 \\
\hline $\begin{array}{l}\text { capacitación sobre abuso se- b) No } \\
\text { xual infantil? }\end{array}$ & 48,2 & 83,1 & 67,6 & 33,3 & 66,7 \\
\hline
\end{tabular}

De acuerdo a las tablas III y IV, sobre un 80\% de los funcionarios, correspondiente a PSS, PIE y DD conoce las consecuencias legales de abusar sexualmente de un/a menor, así mismo, DG y $\mathrm{AD}$, entre un $59,4 \%$ y $75 \%$, indican conocerlas. La decisión de denunciar ASI se toma (58,3\%81,3\%) centrado en el bienestar del niño/a. Destacando que el 33\% de PSS centran su decisión en la «obligación legal», siendo el más alto en comparación con los otros grupos de funcionarios respecto de esta alternativa. Del total de funcionarios, entre un $82,7 \%$ y un $92,6 \%$, frente a la sospecha de ASI señalan que realizarían la denuncia.

Respecto a la pregunta, «conoce a alguna víctima de ASI», entre un 66,2\% y un $83,3 \%$, responde afirmativamente, siendo destacable que DC sea el grupo más alto con un 83,3\%, seguido por profesionales PIE con un 81,3\%, pero sólo un $15 \%$ y un $22,9 \%$, respectivamente, ha realizado una denuncia. La mayoría de los funcionarios (entre un 77,1\% y un 87,7\%) no ha realizado denuncia de ASI. No obstante el 50\% de PSS sí 
la ha realizado. En lo que refiere a capacitación sobre ASI, se observan diferencias según roles y cargo; menor capacitación reciben los $\mathrm{AD}$ con un $16.9 \%$, quién más ha recibido es PSS con 66,7\% (pero manteniendo el 33,3\% sin capacitación), seguido por los DD con un 51,8\%, PIE 33,3\% y DC $32,4 \%$ que han recibido capacitación. Es relevante que tanto DC como PIE, entre un 66 y un $67 \%$ no cuenten con capacitación. Respecto a la efectividad en la aplicación de programas preventivos de ASI, la mayoría da respuesta positiva, $62,3 \%$.

Percepción de aspectos de dominio a nivel psicológico (cargos DC, PSS, PIE).

\section{Tabla V}

\section{Dominios de Nivel Psicológico}

\begin{tabular}{|c|c|c|c|c|}
\hline Ítems & Respuestas & $\begin{array}{l}\text { PIE } \\
(\%)\end{array}$ & $\begin{array}{l}\text { Psicosocial } \\
\quad(\%)\end{array}$ & $\begin{array}{l}\text { Docentes } \\
(\%)\end{array}$ \\
\hline \multirow{2}{*}{$\begin{array}{l}\text { P.7) ¿Estás familiarizado con algún método de evaluación } \\
\text { de abuso sexual infantil? }\end{array}$} & a) Sí & 25,0 & 66,7 & 18,7 \\
\hline & b) $\mathrm{No}$ & 75,0 & 33,3 & 81,3 \\
\hline \multirow{2}{*}{$\begin{array}{l}\text { K.13) El testimonio de un niño es más creíble que el de } \\
\text { un adulto }\end{array}$} & a) Verdadero & 80,9 & 75,0 & 82,8 \\
\hline & b) $\mathrm{Fa}$ & 19,1 & 25, & 17,2 \\
\hline \multirow{2}{*}{$\begin{array}{l}\text { K.15) Si un infante cercano a nosotros sufriera de abuso, } \\
\text { lo sabríamos }\end{array}$} & a) Verdadero & 55,3 & 75,0 & 68,7 \\
\hline & b) Falso & 44,7 & 25,0 & 31,3 \\
\hline \multirow{2}{*}{$\begin{array}{l}\text { K.16) Los abusadores de niños no pueden controlar sus } \\
\text { impulsos sexuales }\end{array}$} & a) Verdadero & 54,3 & 58,3 & 63,4 \\
\hline & b) $\mathrm{Fal}$ & 45,7 & 41,7 & 36,6 \\
\hline \multirow{2}{*}{$\begin{array}{l}\text { K.17) Los abusadores de niños tienen trastornos menta- } \\
\text { les, son enfermos mentales o personas con un alto nivel } \\
\text { de desequilibrio }\end{array}$} & a) $\mathrm{Ve}$ & 70,8 & 58,3 & 71,7 \\
\hline & b) Falso & 29,2 & 41,7 & 28,3 \\
\hline \multirow{2}{*}{$\begin{array}{l}\text { K.18) Algunos abusadores tienen la misma edad que la } \\
\text { víctima }\end{array}$} & a) Ver & 62,5 & 58 & 55,2 \\
\hline & b) $\mathrm{Fal}$ & 37,5 & 41,7 & 44,8 \\
\hline \multirow{2}{*}{$\begin{array}{l}\text { K.19) Los infantes que han sido víctimas, son responsa- } \\
\text { bles de lo que les sucede, ya que pueden prevenirlo }\end{array}$} & a) $\mathrm{Ve}$ & 0 & 0 & 5,4 \\
\hline & b) $\mathrm{Fal}$ & 100 & 100 & 94,6 \\
\hline \multirow{2}{*}{$\begin{array}{l}\text { K.20) A menudo, los infantes inventan haber sido vícti- } \\
\text { mas de abuso sexual, ya que, a veces, son influenciados } \\
\text { por un adulto }\end{array}$} & a) Verdadero & 20,8 & 0 & 22,0 \\
\hline & b) Falso & 79,2 & 100 & 78,0 \\
\hline \multirow{2}{*}{$\begin{array}{l}\text { K.21) Un alto porcentaje de abuso sexual infantil incluye } \\
\text { alguna forma de violencia }\end{array}$} & a) Verdadero & 91,7 & 66,7 & 90,0 \\
\hline & b) Falso & 8,3 & 33,3 & 10,0 \\
\hline \multirow{2}{*}{$\begin{array}{l}\text { K.23) Más de la mitad de los informes sobre abuso sexual } \\
\text { infantil son falsos }\end{array}$} & a) Verdadero & 14,6 & $0 \%$ & 10,5 \\
\hline & b) Falso & 85,4 & $100 \%$ & 89,5 \\
\hline
\end{tabular}

De los 3 grupos de profesionales de trabajo directo con NNA, solo PSS cuenta con el mayor índice de conocimiento con métodos de evaluación de
ASI, aun cuando solo alcanza al 66,7\%, siendo PIE los menos familiarizados con un 25\%, estos mismos cuentan con el indicador más bajo res- 
pecto a reconocer la posibilidad de abuso en un infante cercano con relación a los otros profesionales, con un 55,3\%.

La mayoría de profesionales entre un 54,3\% y un $63,4 \%$, asocia la conducta abusiva sexual a la imposibilidad de ser controlada, siendo significativo que entre el $36,6 \%$ y $45,7 \%$ no consideren esta característica como propia del abusador, y al mismo tiempo una mayoría importante entre un $58,3 \%$ a un $71,7 \%$, visualiza a los abusadores como sujetos «mentalmente enfermos».

La mayoría de los profesionales entre un 75\% y un $82,8 \%$, reconoce en los infantes la credibilidad en sus testimonios, además entre un 79,2\% y un $100 \%$, descartan la posibilidad de que los relatos de los infantes sean a menudo inventados por influencia de algún adulto, y que los informes sobre ASI sean falsos, entre un $85,4 \%$ y un $100 \%$, aun así llama la atención que alrededor de un $20 \%$ de los profesionales indique grados de falta de confianza en la experiencia de abuso en los infantes. Por otro lado, se reconoce que los infantes no tienen responsabilidad alguna del abuso, $(94,6 \%$ a un $100 \%)$ y que este acto incluye alguna forma de violencia, entre un 66,7 y un $91,7 \%$. Una mayoría moderada, entre un 55,2\% y un $62,5 \%$, considera la posibilidad de que el abuso sea también ejercido por un NNA.

\section{Sobre protección al infante.}

Corresponden a Directivos, Administrativos, Docentes, Psicosocial y PIE.

\section{Tabla VI}

Tabla Cruzada entre P8 y C1

\begin{tabular}{ccccc}
\hline & & \multicolumn{3}{c}{ ¿Conoces a alguien que haya sido víctima de abuso sexual? } \\
\hline & & Sí & No & Total \\
\hline Ha realizado alguna & Sí & $22,7 \%$ & $1,4 \%$ & $17,2 \%$ \\
denuncia por abuso & No & $77,3 \%$ & $98,6 \%$ & $82,8 \%$ \\
sexual infantil & Total & $100,0 \%$ & $100,0 \%$ & $100,0 \%$ \\
\hline
\end{tabular}

Tabla VII

Tabla Cruzada entre P10 y C1

\begin{tabular}{ccccc}
\hline & & \multicolumn{3}{c}{$\begin{array}{c}\text { Si solo existieran sospechas de un abuso sexual, ¿̇lo } \\
\text { denunciarías a las autoridades? }\end{array}$} \\
\hline & & Sí & No & Total \\
\hline Ha realizado alguna & Sí & $17,6 \%$ & $14,3 \%$ & $17,1 \%$ \\
denuncia por abuso & No & $82,4 \%$ & $85,7 \%$ & $82,9 \%$ \\
sexual infantil & Total & $100,0 \%$ & $100,0 \%$ & $100,0 \%$ \\
\hline
\end{tabular}


En la tabla VI, se observa que, conociendo a víctimas, el 77,3\% del personal no genera denuncias, frente al 22,7\% que sí lo ha llevado a cabo.

Según la tabla VII, de los sujetos que declaran tener la intención de denunciar bajo sospecha, un $82,4 \%$ no ha realizado denuncia alguna, mientras que sólo un 17,6\% sí lo ha realizado bajo la misma variable.
Finalmente, en la tabla VIII, el 64,8\% de quienes han realizado una denuncia, lo hicieron bajo la importancia del bienestar del infante, el 18,7\% identificó obligaciones éticas y morales y un 16,5\% identifica obligaciones legales ante la seguridad de ocurrencia de un hecho de abuso.

Las tablas IX y X correspondientes a PIE, Psicosocial y Docentes.

Tabla VIII

Tabla Cruzada entre C1 y P9

\begin{tabular}{|c|c|c|c|}
\hline & \multicolumn{3}{|c|}{ Ha realizado alguna denuncia por abuso sexual infantil } \\
\hline & Sí & No & Total \\
\hline $\begin{array}{l}\text { Si estuvieras seguro La importancia del } \\
\text { de que ha ocurri- Bienestar del infante }\end{array}$ & $64,8 \%$ & $77,2 \%$ & $75,0 \%$ \\
\hline $\begin{array}{l}\text { do abuso sexual; lo La obligación ética y } \\
\text { denunciarías, prin- moral }\end{array}$ & $18,7 \%$ & $14,4 \%$ & $15,2 \%$ \\
\hline cipalmente, basado La obligación legal & $16,5 \%$ & $8,4 \%$ & $9,8 \%$ \\
\hline Total & $100,0 \%$ & $100,0 \%$ & $100,0 \%$ \\
\hline
\end{tabular}

Tabla IX

Tabla Cruzada entre P10, P7 y K15

\begin{tabular}{|c|c|c|c|c|}
\hline & & \multicolumn{3}{|c|}{$\begin{array}{c}\text { Si solo existieran sospechas de un abuso sexual, } \\
\text { ¿lo denunciarías a las autoridades? }\end{array}$} \\
\hline & & Sí & No & Total \\
\hline \multirow{3}{*}{$\begin{array}{l}\text { ¿Estás familiarizado } \\
\text { con algún método de } \\
\text { evaluación de abuso } \\
\text { sexual infantil? }\end{array}$} & Sí & $21,8 \%$ & $15,2 \%$ & $20,7 \%$ \\
\hline & No & $78,3 \%$ & $84,8 \%$ & $79,3 \%$ \\
\hline & Total & $100,0 \%$ & $100,0 \%$ & $100,0 \%$ \\
\hline \multirow{3}{*}{$\begin{array}{l}\text { Si un infante cercano } \\
\text { a nosotros sufriera de } \\
\text { abuso, lo sabríamos }\end{array}$} & Verdadero & $68,7 \%$ & $61,5 \%$ & $67,5 \%$ \\
\hline & Falso & $31,3 \%$ & $38,5 \%$ & $32,5 \%$ \\
\hline & Total & $100,0 \%$ & $100,0 \%$ & $100,0 \%$ \\
\hline
\end{tabular}

Según la tabla IX, a pesar de tener intenciones de realizar denuncias bajo sospecha, el 78,3\% no está familiarizado con métodos de evaluación, en contraste con el 21,8\% que respondieron sí en ambas variables. Además, aun cuando la mayoría señala no conocer métodos de evaluación, el $68,7 \%$ sabría si un infante cercano sufre de abuso y realizaría la denuncia bajo sospecha del hecho. 
Tabla X

Tabla Cruzada entre K15 y C1

\begin{tabular}{ccccc}
\hline & & \multicolumn{3}{c}{ Si un infante cercano a nosotros sufriera de abuso, } \\
& & \multicolumn{3}{c}{ lo sabríamos } \\
\hline & & Verdadero & Falso & Total \\
Ha realizado alguna denuncia & Sí & $18,4 \%$ & $15,3 \%$ & $17,4 \%$ \\
por abuso sexual infantil & No & $81,6 \%$ & $84,7 \%$ & $82,6 \%$ \\
& Total & $100,0 \%$ & $100,0 \%$ & $100,0 \%$ \\
\hline
\end{tabular}

Según la tabla X, el 81,6\% no ha realizado alguna denuncia a pesar de que identificarían si un infante cercano sufriera de abuso, en contraste al $18,4 \%$ que si lo ha hecho.

\section{Discusión y conclusiones}

Este estudio ofrece diferencias con lo que señala Márquez et al. (2017), una de ellas es, respecto del género del agresor, en Márquez el 79,3\% afirma que es masculino, mientras que en esta investigación, el 63,4\% afirma que ocurre en ambos sexos por igual. Por otra parte, el $62 \%$ desconoce las consecuencias legales de abusar de un menor de edad, en cambio, en este estudio, el 75\% afirma conocerlas. Finalmente, el $63,3 \%$ señala no conocer a una víctima de ASI, mientras que el $70 \%$ de los sujetos de este estudio, afirman conocer a una víctima.

En los resultados de este estudio asociado a conocimiento básico, el 85,3\% del personal afirma que el ASI comienza a cualquier edad, al contrario de lo hallado en Cantón-Cortés (2013), que establece que los abusos comienzan entre los 8 y los 13 años. Además, el 81,3\% afirma que sucede en ambos sexos por igual, mientras que autores como Bravo y Meléndez (2016); Rúa, Pérez y González (2018); Márquez et al. (2017), hacen hincapié en que ocurre, principalmente, en el sexo femenino. También, el 63,6\% afirma que el agresor tiene las mismas posibilidades de ser mujer u hombre, empero Bravo y Meléndez señalan que el $97 \%$ de los abusadores son hombres.

Asociado a la dimensión del quehacer educativo, existe desigualdad entre los cargos o equipo de trabajo al cual se pertenezca, con relación a la participación en capacitaciones que aborden sexualidad y ASI, siendo, además, muy pocos quienes cuentan con esta formación que permitirían fortalecer la tarea preventiva y de protección, pues resulta importante que cuenten con conocimiento, por ejemplo, sobre factores y/o señales asociadas al ASI fundamentales de identificar, como la hipersexualización, alteración del ciclo del sueño y la depresión mayor (Franco y Ramírez, 2015); factores de riesgo sociofamiliares (Apraez, 2015), hacinamiento, la falta de intimidad, sociedades autoritarias, violentas y la poca visualización de la infancia. Esto es relevante de considerar pues el infante puede no presentar síntomas físicos o psicológicos (Cantón-Cortés y Rosario, 2015) y descubrirse solo por la develación, que se da en el 51\% de los casos (Pou et al., 2001), por tanto, el personal debe estar capaci- 
tado para identificar factores de riesgo, cambios conductuales significativos y recibir una develación en cualquier momento.

Sobre aspectos psicológicos, la principal problemática es el poco dominio teórico y práctico, asociado por ejemplo, al bajo conocimiento de métodos de detección considerando que en el estudio de Gallagher, Badford y Pease (como se citó en Cantón-Cortés, 2013), descubrieron que el $50 \%$ de los casos de ASI son cometidos por niños o adolescentes. Por otro lado, la visualización del agresor como alguien descontrolado y enfermo, tomando en cuenta que la mayoría de los sucesos, según Bravo y Meléndez (2016), en el $57 \%$ de los casos de ASI, se utiliza el soborno o el engaño, por lo tanto, el agresor sería una persona que controla y planifica aquello que va a hacer.

Un hallazgo importante, asociado a la protección de la infancia, es evidenciar que gran parte del personal de establecimientos educativos, conociendo casos de ASI no hacen las denuncias correspondientes aún cuando, contrariamente señalan que lo harían aunque solo tuviera sospechas $(86,5 \%)$ y que identificaría a la víctima si estuviera en su entorno cercano $(68,7 \%)$, es decir impera la abstención de la denuncia sin importar el cargo que se desempeñe, disminuyendo la capacidad de atención temprana y vulnerando los derechos legales de los infantes a pesar del deber ético y legal que esto implica, pues según la legislación chilena, en el artículo 175 del Código Procesal Penal, los directores, docentes e inspectores tienen la obligación de denunciar el suceso, durante las 24 horas posteriores a la develación del hecho.

Por otra parte, se evidencia que profesionales que trabajan con la población escolar más vulnerable, que considera también a niños y niñas en condición de discapacidad (PIE), presenta mayo- res falencias en la temática, aun cuando existen herramientas técnicas para su detección y abordaje, el 55\% no podría reconocer a un infante víctima, pero al mismo tiempo son el equipo que conoce más víctimas y que menos capacitación recibe.

Según los resultados, se evidencia la necesidad de avanzar en políticas públicas que garanticen los derechos de la infancia, promoviendo el rol educativo y protector de los adultos responsables del cuidado, formación y desarrollo de niños, niñas y adolescentes, mediante capacitaciones sobre ASI, dirigidas al personal de los establecimientos educacionales, las que según este estudio, representan sólo un 33,2\%. Es fundamental preparar a agentes educativos en sexualidad y vulneración de derechos, primordial para prevenir y afrontar el ASI, favoreciendo la develación temprana y promoviendo el manejo desde aspectos psicológicos (prevención y reparación), legales (denuncia) y educativos (preventivos). Por ello, se hace imperativo implementar una educación sexual y afectiva con perspectiva de género, pues de acuerdo a la evidencia, reduciría el ASI e impactaría en la gesta de relaciones basadas en el respeto.

Para futuras investigaciones, se sugiere profundizar desde un enfoque cualitativo permitiendo explicar posibles causas o conductas asociadas a esta forma de percibir el fenómeno. Por otra parte, es importante avanzar en la generación y validación de instrumentos que midan conocimiento, creencias y prácticas, asociadas al ASI de modo más específico, contemplando mayores elementos y dimensiones.

Los autores no declaran conflictos de intereses que puedan afectar los resultados de este estudio.

Los autores declaran no haber recibido financiamiento para este artículo. 


\section{Referencias}

Apraez Villamarín, Genny. «Factores de riesgo de abuso sexual infantil». Colombia Forense 2 (2015): 87-94, https://doi.org/10.16925/cf. v3i1.1179

Baita, Sandra and Paula Moreno. Abuso sexual infantil. Cuestiones relevantes para su tratamiento en la justica. Uruguay: UNICEF, 2015.

Bravo, Leslie and Yira Meléndez. «Caracterización del abuso sexual infantil a partir de historias clínicas». Avances en Psicología 24 (2017): 135147, https://doi.org/10.33539/avpsicol.2016. v24n2.149

Cantón, David and María Rosario. «Consecuencias del abuso sexual infantil: una revisión de las variables intervinientes». Anales de Psicología 31 (2015): 552-561, https://doi.org/10.6018/analesps.31.2.180771

Cantón, David. «Prevalencia y características de los abusos sexuales a niños». Archivos de Criminología, Criminalística y Seguridad Privada 13 (2013).

Chile. Código Procesal Penal. Santiago: Editorial Jurídica de Chile, 2018.

Contreras, Lorena, David Huepe and Gorka Navarrete. «Magnitude and characteristics of revictimization through child sexual abuse in Chile». Legal and Criminological Psychology 25 (2020): 33-46, https://doi.org/10.1111/lcrp.12158

Fiscalía Nacional. «Boletín Estadístico Anual Año 2018». Ministerio Público de Chile, http:// www.fiscaliadechile.cl/Fiscalia/estadisticas/index.do

Fondo de las Naciones Unidas para la Infancia. «Una situación habitual: Violencia en las vidas de los niños y los adolescentes». UNICEF, ht- tps://www.unicef.es/publicacion/violencia-enlas-vidas-de-los-ninos-y-los-adolescentes

Franco, Alvaro and Luis Ramírez. «Abuso sexual infantil: perspectiva clínica y dilemas éticos-legales». Revista Colombiana de Psiquiatría 45 (2015): 51-58, https://doi.org/10.1016/j. rcp.2015.07.003

Giménez, Vicenta and Noelia Valladolid. «Detección de abuso sexual en una muestra de donantes de gametos en España». Medicina Reproductiva y Embriología Clínica 4 (2017): 32-44, https://doi.org/10.1016/j.medre.2017.02.002

Gutiérrez, Carolina, Mónica Steinberg and Claudia Capella. «Develación de las agresiones sexuales: estudio de caracterización de niños, niñas y adolescentes». Psykhe 25 (2016): 1-15, https://doi.org/10.7764/psykhe.25.2.852

Hernández, Roberto, Carlos Fernández and María del Pilar Baptista. Metodología de la investigación. México: McGraw-Hill, 2014.

Losada, Analia. «Epidemiología del abuso sexual infantil». Revista de Psicología GEPU 3 (2012): 201-229.

Márquez, María, Verónica Márquez and Genoveva Granados. «Teachers' knowledge and beliefs about child sexual abuse». Journal of Child Sexual Abuse 25 (2016): 538-555, https://doi.or $\mathrm{g} / 10.1080 / 10538712.2016 .1189474$

Martinic, Sergio, «El tiempo y el aprendizaje escolar». Revista Brasileira de Educação 20 (2015): 479-499, doi: 10.1590/s1413-24782015206110

Mazi, Laura and Carolina Asensio. «Abuso sexual infantil: ¿qué signfica escuchar al niño/a?». Familias y Terapias 39 (2015): 41-49. 
Oyanedel, Juan and Harry Ortúzar. «Sistematización de una experiencia piloto de implementación de una sala gessell para la entrevista de niños en un tribunal de familia». Revista Chilena de Pediatría 89 (2018): 649-700, https://doi. org/10.4067/S0370-4106201800500081

Pereda, Noemí and Judit Abad. «Enfoque multidisciplinar de la exploración del abuso sexual infantil». Revista Española de Medicina Legal 39 (2012).: 19-25, doi: 10.1016/j.reml.2012.10.002

Pereda, Noemí and Laura Sicilia. «Reacciones sociales ante la revelación del abuso sexual infantil y malestar psicológico en mujeres víctimas». Psychosocial Intervention 26 (2017): 131-138, https://doi.org/10.1016/j.psi.2017.02.002

Pinto, Gristián and Cristóbal Guerra. «Victimización sexual de niños, niñas y adolescentes chilenos: prevalencia y características asociadas». Revista de Psicología 28 (2019): 1-12, https:// doi.org/10.5354/07 19-0581.2020.55658

Rodríguez, José and Jordi Vallderiola. Metodología de la investigación. Cataluña: Universitat Oberta de Catalunya, 2012

Rúa, Rosa, Violeta Pérez and Rubén González. «El abuso sexual infantil: opinión de los/as profesionales en contextos educativos». Prisma Social 23 (2018): 46-45.

Villanueva, Ibeth. «El abuso sexual infantil: perfil del abusador, la familia, el niño víctima y consecuencias psíquicas del abuso». Psicogente 16 (2013): 451-470.

\section{Contribución de autoría}

Sebastián Araya: Investigación - Análisis Metodología - Redacción, revisión y edición.

Juan Díaz: Investigación - Análisis - Metodología - Redacción, revisión y edición.

María de los Angeles Valenzuela: Investigación - Análisis - Metodología Redacción, revisión y edición.

Karina Tejada: Investigación - Análisis Metodología - Redacción, revisión y edición. 